\title{
Liver Extraction Method from Magnetic Resonance Cholangio-Pancreatography (MRCP) Images
}

\author{
Sajid Ur Rehman Khattak ${ }^{1}$, Dr. Mushtaq $\mathrm{Ali}^{2}$, Faqir Gul ${ }^{3}$, Nadir Hussian Khan ${ }^{4}$, Amanullah Baloch ${ }^{5}$, M. Shoaib \\ Ahmed $^{6}$ \\ Department of Information Technology \\ Hazara University, Mansehra, \\ KPK, Pakistan
}

\begin{abstract}
Liver extraction from medical images like CT scan and MR images is a challenging task. There are many manuals, Semi-automatic and automatic methods available to extract the liver from computerized tomography (CT) scan images and MR images. However, no method is available in the literature to extract the liver from Magnetic Resonance Cholangiopancreatography (MRCP) images. Extracting the liver accurately from MRCP images is needed, so that the physician can diagnose the disease easily and plan preoperative liver surgery accordingly. In this paper, we propose a liver extraction method based on Graph Cut algorithm for liver extraction from MRCP images. The experimental results show that the proposed method is very effective for liver extraction from MRCP images.
\end{abstract}

Keywords-Liver extraction; graph cut algorithm; MRCP images; liver surgery; medical images; liver mask; adaptive thresholding method

\section{INTRODUCTION}

The healthy body depends on the healthy liver. There are many metabolic functions that performed by the liver for the smooth working of other organs. The unhealthy liver causes different types of diseases like obstruction in vessel, tumor, which need surgery. The physician recommends MRCP imaging test to the liver patients. The MRCP is an advance technology of the MRI technology the MRCP images are noninvasive, inexpensive and do not use any ionizing radiation as compare to $\mathrm{CT}$ images [1]. Due to the rapidly increase in the use of MRCP imaging technology an accurate and efficient liver extraction method from MRCP images is needed. An accurate liver extraction will help the radiologist and surgeon in diagnosing the liver diseases and in the planning of preoperative liver surgery as well.

In the computer science technologies the image processing is the most effective techniques which can be helped in different types of medical images. Liver extraction is very important stage in diagnosing the liver diseases. However, it is very difficult and challenging task to extract the liver. Because the liver is non-rigid shape naturally and the intensity value of heart and kidney is similar to liver [2].

Most of the research work for CT scan and MRI images has been conducted on liver extraction. Different type of method like manual, Semi-automatic and automatic methods are available to extract the liver from CT scan images and MR images [5]-[12]. In this paper, we developed an automatic method for liver extraction from MRCP images.
Because of no research work has been reported in the literature. In order to fill the gap, a liver extraction method from MRCP images is proposed in this paper.

Our proposed algorithm works as follows. The MRCP images are grayscale, noisy and low contrast images. First of all remove the noise and enhanced the image. Secondly, find the intensity level of the liver and detect the largest region then extract the largest region in the image. Finally, create the liver mask and apply mask on the liver image to extract the original liver region.

The sequence of the paper as follows. Section 2 is about literature review. Section 3 is about proposed solution and implementation. Section 4 shows the experimental results and section 5 concludes the paper with future work discussion.

\section{LITERATURE REVIEW}

Most of the physicians adopt manual method in which the liver images are traced by mouse clicking on each slice of CT or MR images. The accuracy of manual method is $100 \%$, but it is time-consuming method [3], [4].

In [5] the authors use the manual method to segment the lever volume from CT scan as shown in Fig. 1. In left lateral having segment II and III, in the caudate lobe having segments I, in left medial having segment IV and right lobe having segments V-VIII, each segment has been traced with the mouse positioning cursor. However, the manual method needs skills and expertise of the physicians.

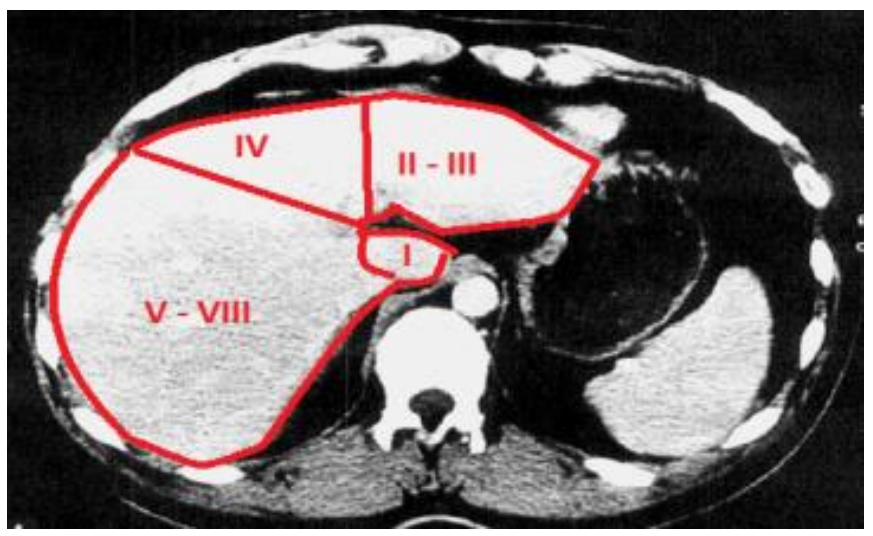

Fig. 1. The Kawasaki Manually Liver Segmentation. 


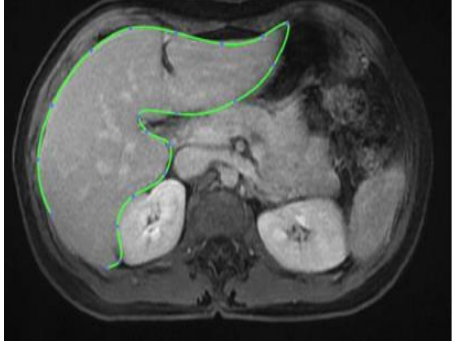

(a)

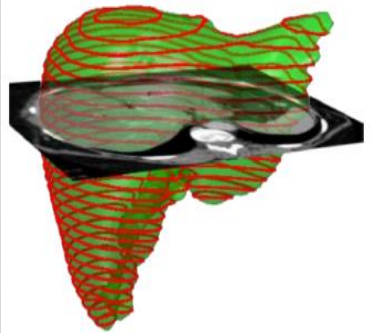

(b)
Fig. 2. Manual Extraction of liver. a) Tracing the Liver Boundaries, b) Obtained the Liver Volume by Summing each Slice.

In [6] the authors give the view on manual liver segmentation, according to author the post-processing software loads the Digital Imaging and Communications in Medicine (DICOM) file of CT or MR image. Then trace the liver boundary by the mouse positioning cursor as shown in Fig. 2 (a). Every pixel enclosed within the boundaries is the cross-sectional areas of the slice. Tracing each slice of CT or MR images and each slice multiplies with the thickness of slice and summing the result of all slices give the liver volume as shown in Fig. 2 (b).

In [7] the authors have proposed a semi-automatic method for liver lesion segmentation from CT scan images. The author does work in the following steps. In the first step, select the CT image slice where the liver is clearly appeared and extract the liver by using the morphological reconstruction. In its applied histogram threshold process to separate the liver, an erosion operation is applied to remove neighboring small parts connected to the liver. In last apply the dilation process to reconstruct the liver. In the second step enhanced the image and improve the image quality and subtract the lesion from liver has been shown in Fig. 3. The experimental results have achieve Sensitivity is $92 \%$ and Specificity $99 \%$.
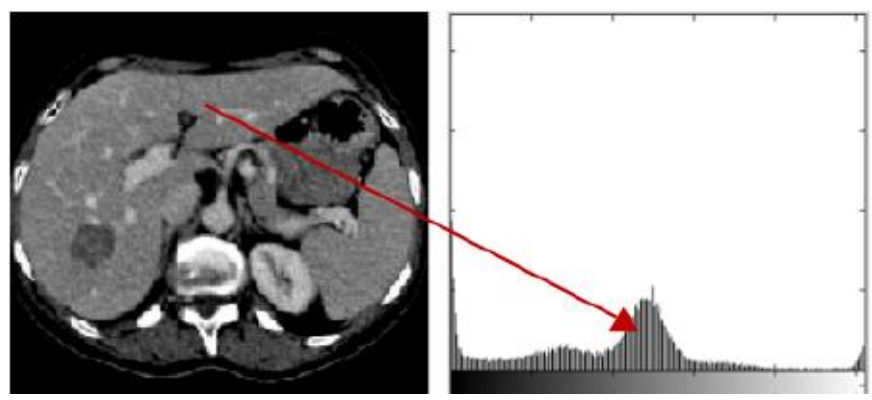

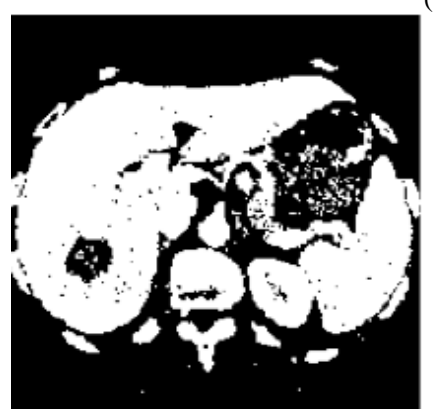

(b)

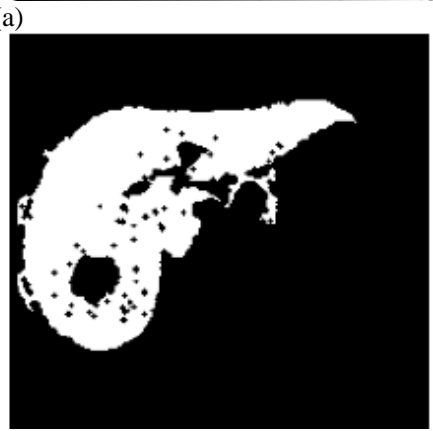

(c)
Fig. 3. Semi-Automatic Method for Liver Extraction. a) Original image, b) Histogram threshold, c) Extraction of liver.
In [8] the authors have described a semi-automatic method for liver extraction from the CT scan, which consists of three steps. In the first step, apply the histogram threshold to find the liver likelihood intensity range. Secondly, region growing method is applied in two sub steps. 1) Using get point function, click on the image where the liver mostly found and got the seed. 2) Giving fixed seed point for an image, but it is a very difficult method by giving fix seed point to each image due to liver shape variation. In the third step, apply the morphological operation like fill the holes, erosion, dilation and median filter to refine the liver image as shown in Fig. 4.

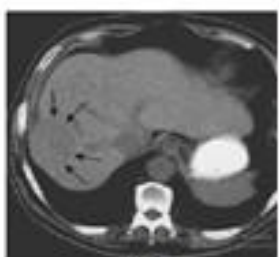

(a)

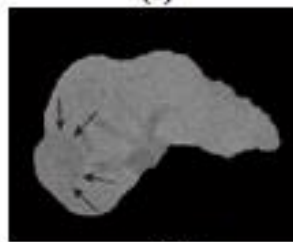

(d)

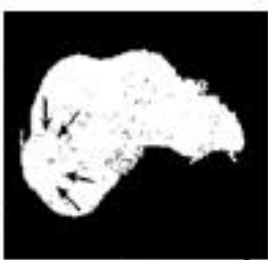

(b)

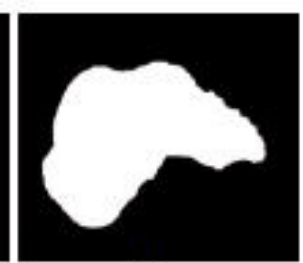

(c)

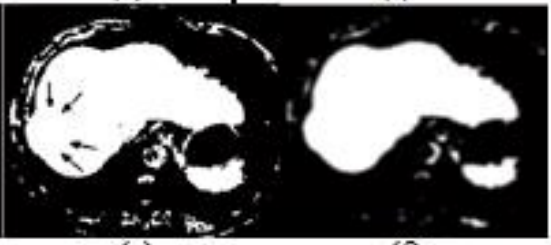

(e)

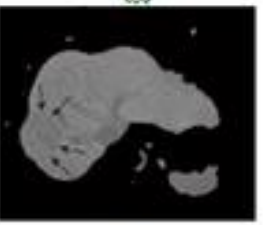

(g)
Fig. 4. Semi-Automatic Liver Extraction from CT Images, a) Original Image, b) Region Growing, c) Morphological Function, d) Extract Liver, e) Median Filter Result, f) Morphological Function, g) Final Result of Liver.

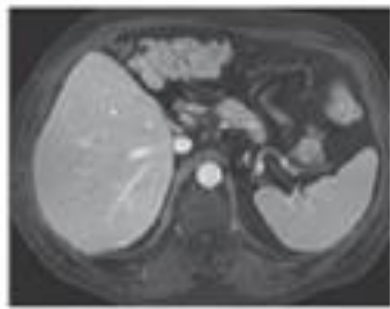

(a)

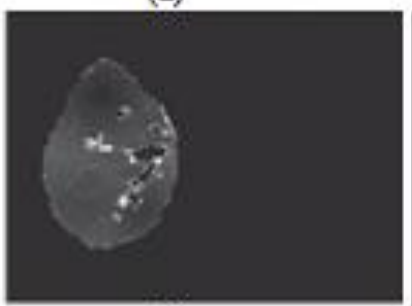

(c)

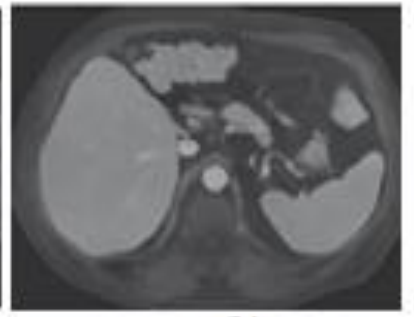

(b)

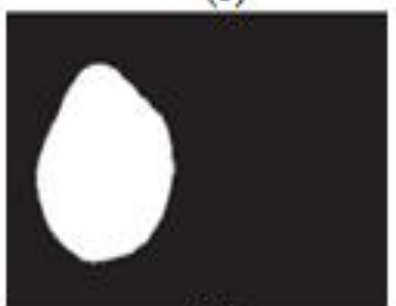

(d)
Fig. 5. Automatic Liver Extraction from MR Images. a) Original Image, b) Noise Removed, c) Scale-Specific Gradient Magnitude, d) Geodesic Active Contour Segmentation. 
In [9] authors have suggested an automatic liver extraction from MR images. The author extracts the liver in four steps. Firstly, apply the anisotropic diffusion filter to remove the noise from image. Secondly, liver boundaries are enhanced with scale-specific gradient magnitude filter. Thirdly, the liver shape is determined by using the fast marching algorithm. In the fourth step, liver shape is refined by using a geodesic active contour level set algorithm as shown in Fig 5. The experimental results show the sensitivity is $92 \%$, specificity $99.8 \%$, and accuracy is $99 \%$. The average computational time is $1.002 \mathrm{~min} /$ case.

In [10] the authors have stated graph cut techniques for liver segmentation from 10 datasets of CT and MR images. The mean shift fitter is applied to remove noise from the original image. Adaptive threshold method is applied for automatic initialization and in the last graph cut technique is applied for liver extraction as shown in Fig 6.

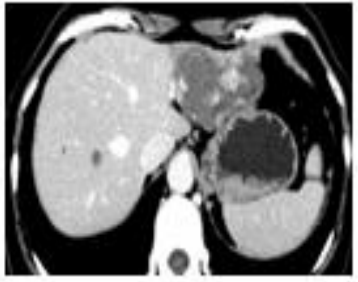

(a)

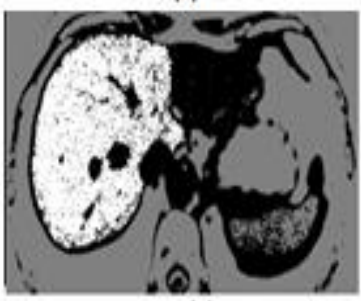

(c)

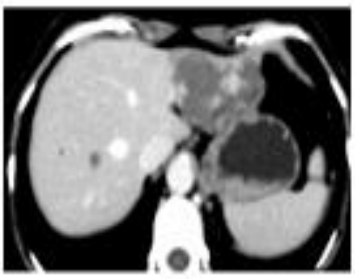

(b)

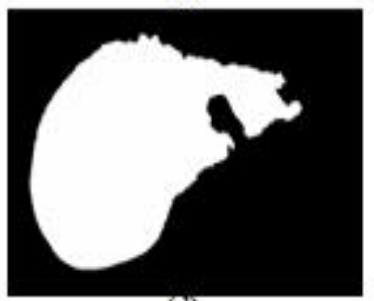

(d)
Fig. 6. Automatic Liver Extraction from CT images. a) Original image, b) Mean shift filter, c) Adaptive threshold method, d) Extract liver.

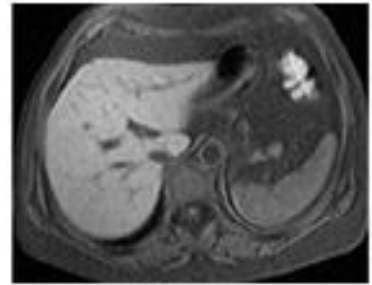

(a)

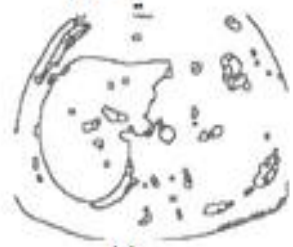

(c)

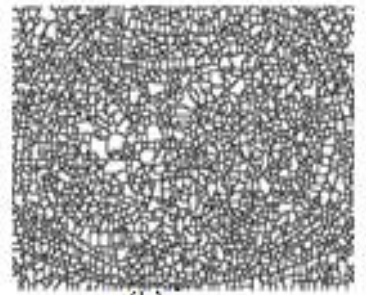

(b)

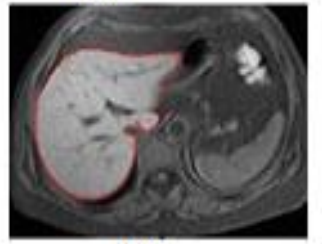

(d)
Fig. 7. Automatic Liver Extraction from MR Images. a) Original Image, b) Watershed transformation, c) Applied proposed algorithms, d) Extracted liver region.
In [11] the authors have proposed an automatic solution for liver extraction from MRI images by using an iterative watershed algorithm and artificial neural network. In the preprocessing step remove the noise from the image by using the gaussian filter and morphological gradients. In next step, liver region is extracted by using an iterative watershed transform and in last apply neural networks to refine the liver image as shown in Fig. 7. The author compared the experimental result with active contour model, the accuracy for the proposed solution is $94 \%$ and for active contour, the method is $92 \%$. The proposed solution is 1.5 times faster the active contour model.

In [12] the authors have surveyed the automatic method for liver segmentation from MR images. The proposed solution is divided into four stages. In the first stage enhances the image by applying image normalization and gamma correction to adjust the image intensity value. Sharp the edges of the image and fill the small holes for the smooth texture. In the second stage, eliminate small objects, extract the large image and perform morphological steps. In the third stage enhances the image applies the gaussian filter the liver is segmented by using the active contour method. In the fourth stage applying morphological erosion and extract object form images by size as shown in Fig. 8. The experimental results show $95 \%$ accuracy.

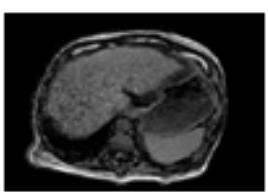

(a)

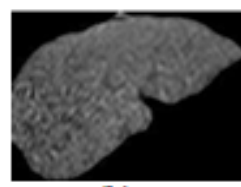

(b)
Fig. 8. Automatic Liver Extraction from MR Images. a) Original image, b) Segmented liver.

\section{LIVER EXTRACTION METHOD}

In this section a liver extraction method for MRCP images presented. Our proposed liver extraction method consists of three main modules namely: 1) Image Pre-processing, 2) Liver Mask Creation and 3) Liver Extraction. The process diagram of liver extraction is shown in Fig 9 and the detail of each of the modules of this method is discussed as follows.

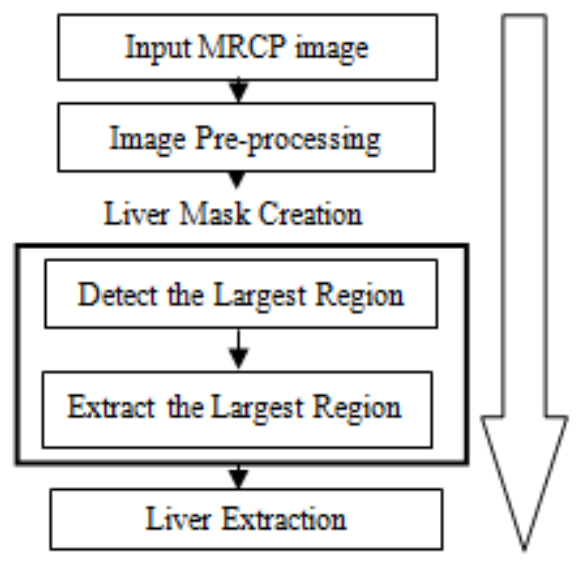

Fig. 9. Process Diagram of Liver Extraction Method. 


\section{A. Image Pre-Processing}

The MRCP images are noisy and low contrast. The aim of this module is to remove noise and smooth the images before extracting the liver from MRCP images. To achieve this aim first of all, the contrast of the original image is enhanced by applying imadjust function. Secondly, the $5 \times$ 5-pixel frame of the Gaussian filters with standard deviation $\sigma=2.5$ is applied on the enhanced image. The output image is enhanced, smoothed and de noised as show in Fig. 10(a-c).

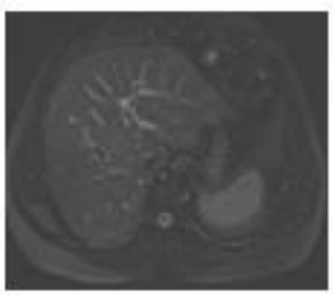

(a)

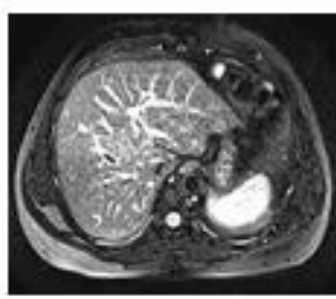

(b)

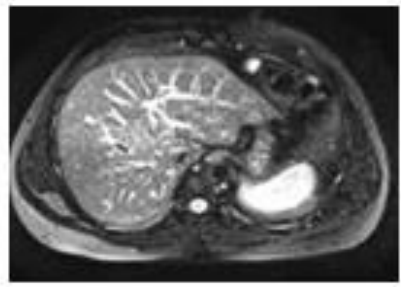

(c)

Fig. 10. The result is generated by applying image pre-processing module. a) Original image, b) Result generated by imadjust function, c) Smoothing and de noising the image applying Gaussian filter.

\section{B. Liver Mask Creation}

This module uses an observation based approach for liver mask creation. The thresholding method, graph cut algorithm and morphological techniques are used for obtaining the liver mask. This module consists of two sub modules namely: 1) Detect the Largest Region and 2) Extract the Largest Region.

1) Detect the Largest Region: The main objective of this sub module is to detect the largest region (Liver). To do this an adaptive thresholding method is applied on image, it is our observation that the range of threshold used for representing the liver region is in the range of $100-210$. Each pixel in the image is compared with fixed minimum and the maximum threshold values. If the image pixel values are less than the minimum range or greater than the maximum range then they are set to 0 , otherwise set to 1 . This threshold is applied on the image Fig. 10 (c) then the output result is get and shown in Fig. 11 (a). Next, bwareaopen morphological function is applied on the resultant image, to remove unwanted small connected components composed of number of pixels less than 5000. The output image is shown in Fig. 11 (b).

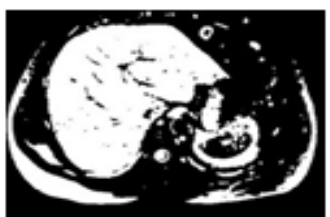

(a)

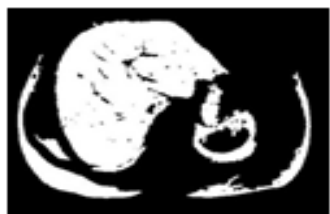

(b)
Fig. 11. The result of detecting largest region. a) Applying threshold, b) Remove small connected components.

2) Extract the Largest Region (Liver): There are many approaches are available that can extract foreground from background. Most of them are based on regional or boundary information which does not achieve the required accuracy. The graph cut algorithm provides both regional and boundary information [13], [14], so the aim of this sub module is extracting the largest region (Liver) by applying the graph cut algorithm. After applying the graph cut algorithm on images Fig. 11 (b), the result of boundary information and regional information as shown in Fig. 12 (a, b).

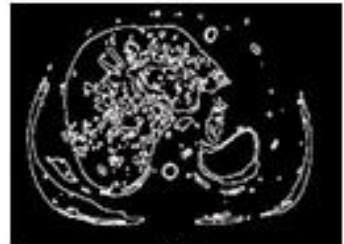

(a)

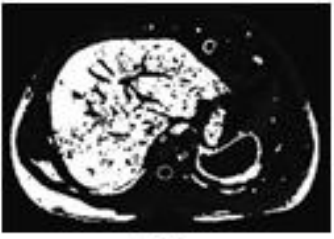

(b)
Fig. 12. Applying the graph cut algorithm for getting (a) The boundary information and (b) regional information.

Then added the regional information as shown in Fig. 12 (a) and boundary information as shown in Fig. 12 (b) then the result as shown in Fig. 13(a) and the imfill function is applied on the resultant image to fill the hole in the image and the output as shown in Fig. 13 (b), Next, bwareaopen morphological function is applied to remove the irrelevant connected components to get the liver image mask as shown in Fig. 13(c).

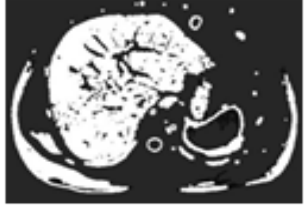

(a)

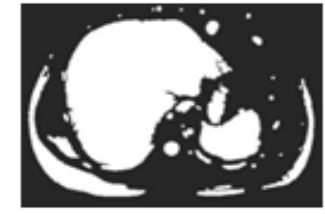

(b)

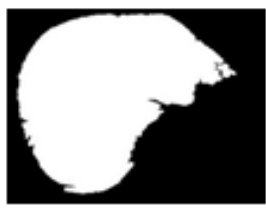

(c)

Fig. 13. The result of extracting largest region. (a) Graph cut algorithm output, (b) Fill the holes, (c) Extracting the liver mask. 


\section{Liver Extraction}

The pixel values of mask generated by the previous module are compared with the original image pixel values. In this module, when the corresponding pixel values of the mask and original image become match as shown in Fig. 14(a, b). Then the values of that pixel is set to 1 , otherwise its values is set to 0 . The extracted liver image is shown in Fig. 14(c).

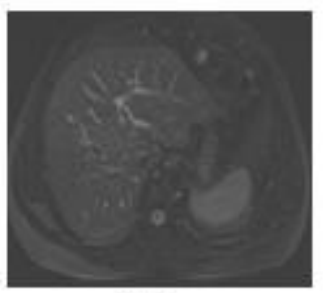

(a)

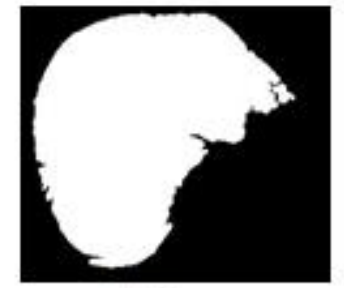

(b)

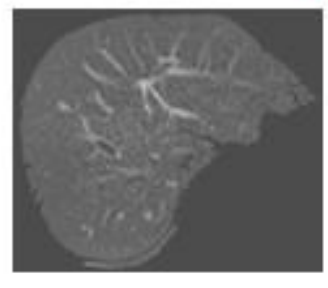

C

Fig. 14. The result of liver extraction is (a) Original image, (b) Apply the liver mask, (c) Extracted liver.

\section{EXPERIMENTAL RESULTS}

In this section evaluation criteria, dataset and results of the proposed method are presented.

\section{A. Evaluation Criteria}

A group of 10 experts included doctors and radiologist examine the result which was generated by our proposed method. However, our proposed method was practically implemented in the clinic on 15 easy, 15 average and 15 difficult test cases. The result was considered correct if liver in the image was fully extracted otherwise the generated result was declared as incorrect.

\section{B. Dataset}

The MRCP images data was not publically available. So the dataset of MRCP images was collected personally in the last four months (Jan-Apr 2018) from 57 patients (37 male patients and 20 female patients) by using the Echelon Oval 1.5T Hitachi machine in Ayub Medical Teaching Hospital Abbottabad, Pakistan. The dataset images in Digital Imaging and Communications in Medicine (DICOM) format and contained 300 MRCP images with different resolution $320 \mathrm{x}$ 320 pixels to $1362 \times 577$ pixels are

\section{Results}

The result of our proposed liver extraction method is shown in Table 1. Our results were evaluated numerically with the help of five parameters namely.

- Overlap error.

- Average Distance.

- Maximum Distance.

- Volume Difference.

- Root Mean square Distance.

TABLE I. RESULT OF OUR PROPOSED LIVER EXTRACTION METHOD FOR 15 CASES

\begin{tabular}{|c|c|c|c|c|c|c|c|c|c|c|c|c|}
\hline \multicolumn{2}{|c|}{ Cases } & \multicolumn{2}{|c|}{$\begin{array}{l}\text { Ovr. Error } \\
(\%) \quad \text { Score }\end{array}$} & \multicolumn{2}{|c|}{$\begin{array}{l}\text { Avg. Dist. } \\
(\mathrm{mm}) \quad \text { Score }\end{array}$} & \multicolumn{2}{|c|}{$\begin{array}{l}\text { Max. Dist. } \\
(\mathrm{mm}) \quad \text { Score }\end{array}$} & \multicolumn{2}{|c|}{$\begin{array}{l}\text { RMS Dist. } \\
(\mathrm{mm}) \quad \text { Score }\end{array}$} & \multicolumn{2}{|c|}{$\begin{array}{l}\text { Vol. Diff. } \\
(\%) \quad \text { Score }\end{array}$} & \multirow{2}{*}{$\begin{array}{l}\text { Total Score } \\
59\end{array}$} \\
\hline 1 & \multirow{5}{*}{ 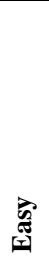 } & 20.1 & 67 & 0.1 & 73 & 30.3 & 32 & 2.4 & 65 & 1.4 & 56 & \\
\hline 2 & & 17.6 & 34 & 1.6 & 43 & 12.4 & 54 & 9.5 & 23 & -5.3 & 45 & 40 \\
\hline 3 & & 19.3 & 23 & 4.3 & 23 & 42.1 & 24 & 3.7 & 67 & 16.4 & 67 & 41 \\
\hline 4 & & 16.0 & 70 & 3.0 & 45 & 20.1 & 56 & 1.9 & 97 & 2.7 & 53 & 64 \\
\hline 5 & & 21.2 & 99 & 5.2 & 78 & 26.7 & 87 & 4.5 & 54 & 0.5 & 98 & 83 \\
\hline 6 & \multirow{5}{*}{ 范 } & 11.5 & 45 & 1.5 & 65 & 56.4 & 43 & 7.8 & 32 & -14.8 & 86 & 54 \\
\hline 7 & & 13.4 & 87 & 3.2 & 73 & 45.8 & 78 & 2.7 & 87 & 4.4 & 54 & 76 \\
\hline 8 & & 18.2 & 74 & 6.2 & 54 & 23.1 & 98 & 9.0 & 44 & 1.9 & 49 & 64 \\
\hline 9 & & 12.9 & 27 & 2.2 & 87 & 11.5 & 42 & 5.6 & 78 & 5.7 & 78 & 62 \\
\hline 10 & & 15.8 & 84 & 3.8 & 42 & 14.7 & 89 & 8.3 & 34 & 0.9 & 92 & 68 \\
\hline 11 & \multirow{5}{*}{ 莺 } & 10.2 & 56 & 0.9 & 87 & 21.3 & 23 & 6.9 & 78 & -18.2 & 45 & 58 \\
\hline 12 & & 14.9 & 40 & 4.5 & 99 & 19.6 & 79 & 1.6 & 86 & 2.5 & 23 & 65 \\
\hline 13 & & 23.6 & 90 & 3.2 & 78 & 42.6 & 35 & 7.4 & 54 & -20.7 & 53 & 62 \\
\hline 14 & & 17.8 & 63 & 2.1 & 46 & 32.1 & 90 & 1.3 & 75 & 8.9 & 68 & 68 \\
\hline 15 & & 5.4 & 14 & 0.1 & 20 & 12.0 & 27 & 4.6 & 25 & 6.4 & 95 & 36 \\
\hline \multicolumn{2}{|c|}{ Average } & 12.06 & 58.2 & 2.8 & 68.6 & 27.38 & 53.8 & 5.1 & 59.9 & -7.3 & 67.9 & 56 \\
\hline
\end{tabular}


Our proposed method is analysis the intensity level of liver. When the intensity value of liver is not laid between the range of 100- 210 then it shows bad output (in cases 2, 6, 13 and 11) in all five parameters otherwise it shows good output (in cases $1,3,4,, 5,7,8,9,10,12,14$, and 15). The performance of the proposed liver extraction method depends on the five parameters. The total average score 89 and the average liver extraction time is 67 seconds (min 23 seconds, max 145 seconds) which is much better than manual extraction.

However, our proposed algorithm had successfully extracted liver from 273 out of $300 \mathrm{MRCP}$ images the average accuracy of our proposed algorithm was $91 \%$.

\section{CONCLUSION AND DISCUSSION}

The literature is not available for liver extraction from MRCP images. But there is a lot of literature is available for liver extraction form CT and MR images. So with the help of above literature review, we are motivated and introduced the new idea for liver extraction from MRCP images due to rapidly increasing in MRCP technologies. In this paper, we proposed new and automatic method for liver extraction from MRCP images. Our proposed method consists on the following steps. Firstly, threshold is applied on the preprocessing step to find the liver intensity range. Secondly, the largest object in the image is extracted. Thirdly, the foreground from the background is separated and a mask is generated. Finally, the mask is applied on the original MRCP image to extract the liver.

In the future, we will extend our proposed work to extract the biliary tract and segment the liver according to $\mathrm{C}$. Couinaud's liver segmentation theory and label the biliary tract on the segmented parts.

\section{ACKNOWLEDGMENT}

The author would like to Special thank Dr. Nadir Farid Khattak and also Radiologist Muhammad Asif for their expert opinions and providing MRCP data for research.

\section{REFERENCES}

[1] Mehmet Ali, Omer Karahan, "Comparison of Endoscopic Retrograde Cholangiopancreatography (ERCP) and Magnetic Resonance Cholangiopancreatography (MRCP) in Bile Duct Imaging", Scientific Research, Sep 2012.

[2] Mortele, K. J., Cantisani, V., Trosisi, R., "Preoprative Liver Donor Evaluation: Imaging and Pitfalls, Liver Transplantation" Vol 9, No 9, Suppl 1, pp S6-S14, Sep 2003.

[3] Yang Xiaopeng, Jae Do Yang, "Segmentation of Liver and Vessel from CT Images and Classification of Liver Segments for Preoperative Liver Surgical Planning in Living Donor Liver Transplantation", Computer Method and Programs in Biomedicine, May 2018.

[4] Ina Singh, "A Study of Effective Segmentation Technique for Liver Segmentation", International Journal of Advanced Research in Computer Engineering \& Technology (IJARCET), Vol 4, April 2015.

[5] S. Kawasaki, M. Makuuchi "Preoperative Measurement of Segmental Liver Volume of Donor for Living Transplantation", American Association for the Study of Liver Diseases, Jun 1993.

[6] Akshat Gotra, Lojan Sivakumaran, "Review: Liver Segmentation: indication, techniques and future directions", Springer Link, Jun 2017.

[7] Belgherbi, A. Hadjidj, I. "A Semi-Automatic Method for the Liver Lesion Extraction From a CT image Based on Mathematical Morphology", iMedPub Journals, Vol 2, 2013.

[8] Shaimma A. Elmorsy, "A Region Growing Liver Segmentation Method with Advanced Morphological Enhancement”, IEEE, March 2015.

[9] BUI Dinh Tien "Studying Methods of Automatic Liver Segmentation from MRI Images", Semantic Scholar, 2015.

[10] Laurent Massoptier "Fully Automatic Liver Segmentation through Graph-Cut Technique", IEEE Conference paper, August 2007.

[11] Hassan Masoumi, Aliraza Behrad, "Automatic Liver Segmentation in MRI images using an Iterative Watershed Algorithm and Artificial Neural Network", Elsevier, Feb 2012.

[12] Roaa G. Mohamad, Noha A. Seada "Automatic Liver Segmentation from MRI Images using Active Contours", International Journal of Computer Applications (IJCA), Oct 2017.

[13] Y. B. Yuri, G F. Lea, "Graph Cuts and Efficient N-D Images segmentation", International Journal of Computer Vision 2006.

[14] Zeyun Yu. Ming Xu, "Biomedical Image Segmentation via Constrained Graph Cuts and Pre-Segmentation", IEEE, Feb 2012.

\section{AUTHORS' PROFILE}
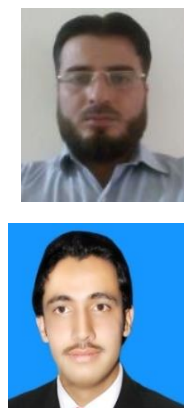

Saiid ur Rehman Khattak received the B.S degree in computer science from Allama Iqbal open (AIOU) University, Islamabad, Pakistan, in 2011. He is currently an MS student in the Department of Information Technology (IT), Hazara University, Dhodial KP, Pakistan. His primary research interests in medical image processing.

Mr. Nadir Hussain received the B.s degree in computer science from Hazra University, Mansehra, KP, Pakistan 2016.He is currently an Ms Student Department of Information Technology Hazra University , Mansehra KP, Pakistan His primary research interests medical image processing. 